\title{
Complex Variable Meshless Manifold Method for Elastic Dynamic Problems
}

\author{
Hongfen Gao ${ }^{1}$ and Gaofeng Wei ${ }^{2}$ \\ ${ }^{1}$ College of Mechanical and Electrical Engineering, Shandong Management University, Jinan 250357, China \\ ${ }^{2}$ School of Mechanical and Automotive Engineering, Qilu University of Technology, Jinan 250353, China \\ Correspondence should be addressed to Hongfen Gao; gaohongfen@126.com
}

Received 7 January 2016; Accepted 28 February 2016

Academic Editor: Elena Benvenuti

Copyright (c) 2016 H. Gao and G. Wei. This is an open access article distributed under the Creative Commons Attribution License, which permits unrestricted use, distribution, and reproduction in any medium, provided the original work is properly cited.

\begin{abstract}
Combining the finite covering technical and complex variable moving least square, the complex variable meshless manifold method can handle the discontinuous problem effectively. In this paper, the complex variable meshless method is applied to solve the problem of elastic dynamics, the complex variable meshless manifold method for dynamics is established, and the corresponding formula is derived. The numerical example shows that the numerical solutions are in good agreement with the analytical solution. The CVMMM for elastic dynamics and the discrete forms are correct and feasible. Compared with the traditional meshless manifold method, the CVMMM has higher accuracy in the same distribution of nodes.
\end{abstract}

\section{Introduction}

The transient analysis is still an important part of the elastic mechanics in the elastic dynamic simulation. Because of the complexity of the problem, the numerical calculation has not been improved $[1,2]$. Wilkins first studied dynamics of embedded wafer crack, elliptical crack, and semi elliptical surface crack with finite difference method (FDM) [3]. Because the finite difference method cannot describe the dynamic singular stress field, the method is not widely used. The finite element method (FEM) can simulate the dynamics problem, but the data preparation and calculation time are more lager.

In recent decades, the boundary element method (BEM) has been developed. It has the advantages of reducing dimension, saving computation time, and solving high accuracy and can deal with the problem of stress concentration and infinite and semi-infinite domains [4]. Therefore, the BEM has shown a greater potential than FEM and has been widely used in the field of elastic mechanics. Currently, the transient analysis of the elastic dynamic problem with BEM is mainly divided into time difference and spatial domain boundary element method, time domain method, and integral transformation method. J. Sládek and V. Sládek used Laplace transform method to study the buried wafer crack of the infinite elastic body under dynamic stress intensity factor of impact load [5]. Fedeliński studied the dynamic stress intensity factors by using the boundary element method [6].

Both the finite element method and the boundary element method are based on the element method. In the process of solving the problem of elastic dynamics, the accuracy of the solution can be influenced by the shape of the element when the element is extremely deformed [7, 8]. In recent years, with the research of the meshless methods, the meshless method is gradually taking a unique advantage in the study of dynamic problems.

There are many meshless methods, such as smoothed particle hydrodynamics method (SPH) $[9,10]$, the element-free Galerkin (EFG) method [11,12], the collocation point method [13], the finite point method (FPM) $[14,15]$, reproducing kernel particle method (RKPM) [16], multiscale reproducing kernel particle method [17], the hp cloud method [18], the partition of unity finite element method $[19,20]$, generalized finite element method [21], meshless local Petrov-Galerkin method [22], least-square collocation meshless method [23], 
meshless weighted least-square method [24], complex variable meshless method (CVMM) [25, 26], and complex variable meshless manifold method (CVMMM) [27].

The CVMM is established by the complex variable moving least-squares (CVMLS) approximation [28, 29]. In the CVMLS approximation, the number of the unknown coefficients in the trial function is less than that of the MLS approximation, and the CVMLS approximation just needs fewer nodes in the domain than the MLS approximation. Then, combining CVMM method with manifold method, complex variable meshless manifold method (CVMMM) was presented and the method has been applied to the two-dimensional elastic problems, the fracture problems, bimaterial problems [30, 31], and so forth. This method has higher computational efficiency and precision than the traditional meshless method. In this paper, the dynamic problem will be researched through the CVMMM.

\section{CVMMM for Elastic Dynamic Problems}

2.1. Fundamental Equations of Elastic Dynamics. Setting the solving domain as $\Omega$, and the boundary as $\Gamma$, the fundamental equation of the two-dimensional linear elastic dynamics is as follows.

(1) Equilibrium equation is

$$
\mathbf{L} \boldsymbol{\sigma}+\mathbf{b}=\rho \ddot{\mathbf{u}}+\mu \dot{\mathbf{u}} \quad(\text { in domain of } \Omega),
$$

where $\mathbf{L}$ is the differential operator matrix,

$$
\mathbf{L}=\left[\begin{array}{cc}
\frac{\partial}{\partial x_{1}} & 0 \\
0 & \frac{\partial}{\partial x_{2}} \\
\frac{\partial}{\partial x_{2}} & \frac{\partial}{\partial x_{1}}
\end{array}\right]
$$

$\boldsymbol{\sigma}$ is the stress of any point $\left(x_{1}, x_{2}\right)$ in the domain $\Omega$,

$$
\boldsymbol{\sigma}^{T}=\left(\sigma_{11}\left(x_{1}, x_{2}, t\right), \sigma_{22}\left(x_{1}, x_{2}, t\right), \sigma_{12}\left(x_{1}, x_{2}, t\right)\right),
$$

b is the body force of any point $\left(x_{1}, x_{2}\right)$ in the domain $\Omega$,

$$
\mathbf{b}^{T}=\left(b_{1}\left(x_{1}, x_{2}, t\right), b_{2}\left(x_{1}, x_{2}, t\right)\right),
$$

$\rho$ is mass density, and $\mu$ is camping coefficient; $\ddot{\mathbf{u}}$ and $\dot{\mathbf{u}}$ represent the two-order derivative and first-order derivative of the displacement to time, respectively, and can be expressed as

$$
\begin{aligned}
& \ddot{\mathbf{u}}^{T}=\left(\ddot{u}_{1}\left(x_{1}, x_{2}, t\right), \ddot{u}_{2}\left(x_{1}, x_{2}, t\right)\right), \\
& \dot{\mathbf{u}}^{T}=\left(\dot{u}_{1}\left(x_{1}, x_{2}, t\right), \dot{u}_{2}\left(x_{1}, x_{2}, t\right)\right) .
\end{aligned}
$$

$\rho \ddot{\mathbf{u}}$ and $\mu \dot{\mathbf{u}}$ represent inertia force and damping force, respectively.

(2) Geometrical equations are

$$
\boldsymbol{\varepsilon}=\mathbf{L} \cdot \mathbf{u} \quad(\text { in domain of } \Omega),
$$

where $\boldsymbol{\varepsilon}$ and $\mathbf{u}$ are the strain and displacement of any point in the domain $\Omega$, respectively, and can be given as

$$
\begin{aligned}
& \boldsymbol{\varepsilon}^{T}=\left(\boldsymbol{\varepsilon}_{11}\left(x_{1}, x_{2}, t\right), \boldsymbol{\varepsilon}_{22}\left(x_{1}, x_{2}, t\right), \boldsymbol{\varepsilon}_{12}\left(x_{1}, x_{2}, t\right)\right), \\
& \mathbf{u}^{T}=\left(u_{1}\left(x_{1}, x_{2}, t\right), u_{2}\left(x_{1}, x_{2}, t\right)\right) .
\end{aligned}
$$

(3) Physical equation is

$$
\boldsymbol{\sigma}=\mathbf{E} \cdot \boldsymbol{\varepsilon},
$$

where $\mathbf{E}$ is elastic matrix.

(4) Boundary conditions are

$$
\begin{aligned}
\mathbf{u} & =\overline{\mathbf{u}} \quad\left(\text { on the displacement boundary } \Gamma_{u}\right), \\
\mathbf{n} \cdot \boldsymbol{\sigma} & =\overline{\mathbf{t}} \quad\left(\text { on the force boundary } \Gamma_{t}\right),
\end{aligned}
$$

where $\overline{\mathbf{u}}$ is the known displacement on the displacement boundary $\Gamma_{u}$, and $\overline{\mathbf{t}}$ is the known surface force on the surface force boundary $\Gamma_{t} . \Gamma=\Gamma_{u} \cup \Gamma_{t}, \Gamma_{u} \cap \Gamma_{t}=\phi$, and $\Gamma$ is the boundary of the domain $\Omega$. Consider

$$
\mathbf{n}=\left[\begin{array}{ccc}
n_{1} & 0 & n_{2} \\
0 & n_{2} & n_{1}
\end{array}\right],
$$

where $n_{1}$ and $n_{2}$ are the direction cosine of the outer normal $N$ for the boundary $\Gamma_{t}$, respectively.

(5) Initial condition is

$$
\begin{aligned}
& \mathbf{u}(z, 0)=\mathbf{u}_{0}(z), \quad z \in \Omega, \\
& \dot{\mathbf{u}}(z, 0)=\mathbf{v}_{0}(z), \quad z \in \Omega,
\end{aligned}
$$

where $\mathbf{u}_{0}(z)$ and $\mathbf{v}_{0}(z)$ are initial displacement and initial velocity of any node $z=x_{1}+i x_{2}$ in the domain $\Omega$.

2.2. Integral Weak Form of Elastic Dynamics. The equivalent integral weak form of elastic dynamics is

$$
\begin{gathered}
\int_{\Omega} \delta \mathbf{u}^{T} \rho \ddot{\mathbf{u}} d \Omega+\int_{\Omega} \delta \mathbf{u}^{T} \mu \dot{\mathbf{u}} d \Omega+\int_{\Omega} \delta \boldsymbol{\varepsilon}^{T} \boldsymbol{\sigma} d \Omega \\
-\int_{\Gamma_{u}} \delta \mathbf{u}^{T} \mathbf{b} d \Gamma-\int_{\Gamma_{t}} \delta \mathbf{u}^{T} \overline{\mathbf{t}} d \Gamma=0 .
\end{gathered}
$$

Substituting geometric equation (6) and physical equation (8) into (12), we have

$$
\begin{aligned}
\int_{\Omega} \delta \mathbf{u}^{T} \rho \ddot{\mathbf{u}} d \Omega+\int_{\Omega} \delta \mathbf{u}^{T} \mu \dot{\mathbf{u}} d \Omega \\
\quad+\int_{\Omega} \delta(\mathbf{L} \mathbf{u})^{T} \mathbf{E}(\mathbf{L} \mathbf{u}) d \Omega-\int_{\Gamma_{u}} \delta \mathbf{u}^{T} \mathbf{b} d \Gamma \\
\quad-\int_{\Gamma_{t}} \delta \mathbf{u}^{T} \overline{\mathbf{t}} d \Gamma=0 .
\end{aligned}
$$


2.3. CVMMM for Elastic Dynamics. The displacement of any node at any time $t$ can be approximated by using node displacement $\mathbf{u}_{I}(t)=\mathbf{u}\left(z_{I}, t\right)$ in the influence domain. For CVMMM, the displacement of any node $z$ at any time $t$ within the domain can be expressed as

$$
\begin{aligned}
\overline{\mathbf{u}}(z, t) & =\mathbf{u}_{1}(z, t)+i \mathbf{u}_{2}(z, t)=\Phi(z) \cdot \overline{\mathbf{U}} \\
& =\sum_{I=1}^{n} \phi_{I}(z) \cdot \bar{u}_{I}(t),
\end{aligned}
$$

where

$$
\begin{aligned}
\overline{\mathbf{U}}^{T} & =\left(\bar{u}_{1}(t), \bar{u}_{2}(t), \ldots, \bar{u}_{n}(t)\right), \\
\bar{u}_{I}(t) & =u_{1}\left(z_{I}, t\right)+i u_{2}\left(z_{I}, t\right) .
\end{aligned}
$$

By (14) and (16), we can obtain

$$
\begin{aligned}
\mathbf{u}(z, t)= & \left(\begin{array}{l}
u_{1}(z, t) \\
u_{2}(z, t)
\end{array}\right) \\
= & \sum_{I=1}^{n}\left[\begin{array}{ll}
\operatorname{Re}\left[\Phi_{I}(z)\right] & -\operatorname{Im}\left[\Phi_{I}(z)\right] \\
\operatorname{Im}\left[\Phi_{I}(z)\right] & \operatorname{Re}\left[\Phi_{I}(z)\right]
\end{array}\right] \\
& \cdot\left(\begin{array}{l}
u_{1}\left(z_{I}, t\right) \\
u_{2}\left(z_{I}, t\right)
\end{array}\right)=\sum_{I=1}^{n} \widetilde{\Phi}_{I}(z) \cdot \mathbf{u}_{I}(t),
\end{aligned}
$$

where

$$
\widetilde{\boldsymbol{\Phi}}(z)=\left[\begin{array}{ll}
\operatorname{Re}\left[\sum_{i=1}^{n} \Phi_{i}(z)\right] & \operatorname{Im}\left[\sum_{i=1}^{n} \Phi_{i}(z)\right] \\
\operatorname{Im}\left[\sum_{i=1}^{n} \Phi_{i}(z)\right] & \operatorname{Re}\left[\sum_{i=1}^{n} \Phi_{i}(z)\right]
\end{array}\right]
$$

and $\mathbf{u}_{I}(t)$ is the displacement of node $z_{I}$ at time $t$; that is,

$$
\mathbf{u}_{I}(t)=\left(u_{1}\left(z_{I}, t\right), u_{2}\left(z_{I}, t\right)\right)^{T}, \quad I=1,2, \ldots, n .
$$

The velocity and acceleration of any node $z$ at any time $t$ within the domain $\Omega$ can be expressed as

$$
\begin{aligned}
& \dot{\mathbf{u}}(z, t)=\left(\dot{u}_{1}(z, t), \dot{u}_{2}(z, t)\right)^{T}=\sum_{I=1}^{n} \widetilde{\boldsymbol{\Phi}}_{I}(z) \cdot \dot{\mathbf{u}}_{I}(t), \\
& \ddot{\mathbf{u}}(z, t)=\left(\ddot{u}_{1}(z, t), \ddot{u}_{2}(z, t)\right)^{T}=\sum_{I=1}^{n} \widetilde{\boldsymbol{\Phi}}_{I}(z) \cdot \ddot{\mathbf{u}}_{I}(t),
\end{aligned}
$$

where $\dot{\mathbf{u}}_{I}(t)$ and $\ddot{\mathbf{u}}_{I}(t)$ are the velocity and acceleration of node $z_{I}$ at time $t$, respectively:

$$
\begin{aligned}
& \dot{\mathbf{u}}_{I}(t)=\left(\dot{u}_{1}\left(z_{I}, t\right), \dot{u}_{2}\left(z_{I}, t\right)\right)^{T}, \\
& \ddot{\mathbf{u}}_{I}(t)=\left(\ddot{u}_{1}\left(z_{I}, t\right), \ddot{u}_{2}\left(z_{I}, t\right)\right)^{T} .
\end{aligned}
$$

From (17), Lu of (13) can be expressed as

$$
\begin{gathered}
\mathbf{L u}=\mathbf{L} \sum_{I=1}^{n} \widetilde{\boldsymbol{\Phi}}_{I} \mathbf{u}_{I}(t)=\sum_{I=1}^{n} \mathbf{L} \widetilde{\boldsymbol{\Phi}}_{I} \mathbf{u}_{I}(t)=\sum_{I=1}^{n}\left[\begin{array}{cc}
\frac{\partial}{\partial x_{1}} & 0 \\
0 & \frac{\partial}{\partial x_{2}} \\
\frac{\partial}{\partial x_{2}} & \frac{\partial}{\partial x_{1}}
\end{array}\right]\left[\begin{array}{cc}
\operatorname{Re}\left[\Phi_{I}\left(z_{I}\right)\right] & -\operatorname{Im}\left[\Phi_{I}\left(z_{I}\right)\right] \\
\operatorname{Im}\left[\Phi_{I}\left(z_{I}\right)\right] & \operatorname{Re}\left[\Phi_{I}\left(z_{I}\right)\right]
\end{array}\right] \cdot \mathbf{u}_{I}(t) \\
=\sum_{I=1}^{n}\left[\begin{array}{cc}
\operatorname{Re}\left[\Phi_{I, 1}\left(z_{I}\right)\right] & -\operatorname{Im}\left[\Phi_{I, 1}\left(z_{I}\right)\right] \\
\operatorname{Im}\left[\Phi_{I, 2}\left(z_{I}\right)\right] & \operatorname{Re}\left[\Phi_{I, 2}\left(z_{I}\right)\right] \\
\operatorname{Re}\left[\Phi_{I, 2}\left(z_{I}\right)\right]+\operatorname{Im}\left[\Phi_{I, 1}\left(z_{I}\right)\right] & \operatorname{Re}\left[\Phi_{I, 1}\left(z_{I}\right)\right]-\operatorname{Im}\left[\Phi_{I, 2}\left(z_{I}\right)\right]
\end{array}\right] \cdot \mathbf{u}_{I}(t)=\sum_{I=1}^{n} \mathbf{B}_{I}\left(z_{I}\right) \mathbf{u}_{I}(t),
\end{gathered}
$$

where $\Phi_{I, 1}\left(z_{I}\right)$ and $\Phi_{I, 2}\left(z_{I}\right)$ are the derivative of the shape function of CVMMM to $x_{1}$ and $x_{2}$, respectively.

Substituting (17), (19), (20), and (23) into (13), we can obtain

$$
\begin{aligned}
\int_{\Omega} \delta & \left(\sum_{I=1}^{n} \widetilde{\boldsymbol{\Phi}}_{I} \mathbf{u}_{I}\right)^{T} \cdot \rho \cdot\left(\sum_{J=1}^{n} \widetilde{\boldsymbol{\Phi}}_{J} \ddot{\mathbf{u}}_{J}\right) d \Omega \\
& +\int_{\Omega} \delta\left(\sum_{I=1}^{n} \widetilde{\boldsymbol{\Phi}}_{I} \mathbf{u}_{I}\right)^{T} \cdot \mu \cdot\left(\sum_{J=1}^{n} \widetilde{\boldsymbol{\Phi}}_{J} \dot{\mathbf{u}}_{J}\right) d \Omega
\end{aligned}
$$

$$
\begin{aligned}
& +\int_{\Omega} \delta\left(\sum_{I=1}^{n} \mathbf{B}_{I} \mathbf{u}_{I}\right)^{T} \cdot \mathbf{E} \cdot\left(\sum_{J=1}^{n} \mathbf{B}_{J} \mathbf{u}_{J}\right) d \Omega \\
& -\int_{\Gamma_{u}} \delta\left(\sum_{I=1}^{n} \widetilde{\boldsymbol{\Phi}}_{I} \mathbf{u}_{I}\right)^{T} \cdot \mathbf{b} d \Gamma-\int_{\Gamma_{t}} \delta\left(\sum_{I=1}^{n} \widetilde{\boldsymbol{\Phi}}_{I} \mathbf{u}_{I}\right)^{T} \\
& \cdot \overline{\mathbf{t}} d \Gamma=0 .
\end{aligned}
$$

Due to arbitrary of variation $\delta \mathbf{u}$ for node displacement, the system of equation can be obtained as follows:

$$
\mathbf{M U ̈}(t)+\mathbf{C} \dot{\mathbf{U}}(t)+\mathbf{K U}(t)=\mathbf{F}(t),
$$


where $\ddot{\mathbf{U}}(t), \dot{\mathbf{U}}(t)$, and $\mathbf{U}(t)$ are the node acceleration vector, velocity vector, and displacement vector of the system, respectively; $\mathbf{M}, \mathbf{C}, \mathbf{K}$, and $\mathbf{F}(t)$ are the mass matrix, damping matrix, stiffness matrix, and load matrix of the system, respectively, and can be expressed as

$$
\begin{aligned}
\ddot{\mathbf{U}} & =\left(\ddot{\mathbf{u}}_{1}^{T}, \ddot{\mathbf{u}}_{2}^{T}, \ldots, \ddot{\mathbf{u}}_{n}^{T}\right)^{T}, \\
\dot{\mathbf{U}} & =\left(\dot{\mathbf{u}}_{1}^{T}, \dot{\mathbf{u}}_{2}^{T}, \ldots, \dot{\mathbf{u}}_{n}^{T}\right)^{T}, \\
\mathbf{U} & =\left(\mathbf{u}_{1}^{T}, \mathbf{u}_{2}^{T}, \ldots, \mathbf{u}_{n}^{T}\right)^{T}, \\
\mathbf{M}_{I J} & =\int_{\Omega} \widetilde{\boldsymbol{\Phi}}_{I}^{T} \cdot \rho \cdot \widetilde{\boldsymbol{\Phi}}_{J} d \Omega, \quad(I, J=1,2, \ldots, n), \\
\mathbf{C}_{I J} & =\int_{\Omega} \widetilde{\boldsymbol{\Phi}}_{I}^{T} \cdot \mu \cdot \widetilde{\boldsymbol{\Phi}}_{J} d \Omega, \quad(I, J=1,2, \ldots, n), \\
\mathbf{K}_{I J} & =\int_{\Omega} \mathbf{B}_{I}^{T} \cdot \mathbf{E} \cdot \mathbf{B}_{J} d \Omega, \quad(I, J=1,2, \ldots, n), \\
\mathbf{F}_{I} & =\int_{\Gamma_{u}} \widetilde{\boldsymbol{\Phi}}_{I}^{T} \cdot \mathbf{b} d \Gamma+\int_{\Gamma_{t}} \widetilde{\boldsymbol{\Phi}}_{I}^{T} \cdot \overline{\mathbf{t}} d \Gamma,
\end{aligned}
$$

2.4. Processing of Boundary Conditions. In this paper, penalty function method is used to deal with displacement boundary condition. A new item $\alpha \int_{\Gamma_{u}} \delta \mathbf{u}^{T} \mathbf{S}(\mathbf{u}-\overline{\mathbf{u}}) d \Gamma$ is added to (13) to compulsorily satisfy the displacement boundary condition. The modified integral weak form can be written as

$$
\begin{aligned}
\int_{\Omega} \delta \mathbf{u}^{T} \rho \ddot{\mathbf{u}} d \Omega+\int_{\Omega} \delta \mathbf{u}^{T} \mu \dot{\mathbf{u}} d \Omega \\
\quad+\int_{\Omega} \delta(\mathbf{L} \mathbf{u})^{T} \mathbf{E}(\mathbf{L} \mathbf{u}) d \Omega-\int_{\Gamma_{u}} \delta \mathbf{u}^{T} \mathbf{b} d \Gamma \\
\quad-\int_{\Gamma_{t}} \delta \mathbf{u}^{T} \overline{\mathbf{t}} d \Gamma+\alpha \int_{\Gamma_{u}} \delta \mathbf{u}^{T} \mathbf{S}(\mathbf{u}-\overline{\mathbf{u}}) d \Gamma=0,
\end{aligned}
$$

where $\alpha$ is the penalty factor, and the value is generally

$$
\begin{aligned}
& \alpha=1.0 \times 10^{5} \sim 1.0 \times 10^{8}, \\
& \mathbf{S}=\left[\begin{array}{ll}
s_{1} & 0 \\
0 & s_{2}
\end{array}\right] .
\end{aligned}
$$

When the direction of $x_{1}$ (or $x_{2}$ ) has a displacement constraint, the value of $s_{1}$ (or $s_{2}$ ) is correspondingly equal to 1 ; otherwise it is 0 .

Substituting (17) into the last item of (28), we have

$$
\begin{aligned}
& \alpha \int_{\Gamma_{u}} \delta \mathbf{u}^{T} \mathbf{S}(\mathbf{u}-\overline{\mathbf{u}}) d \Gamma \\
& \quad=\alpha \int_{\Gamma_{u}} \delta\left(\sum_{I=1}^{n} \widetilde{\boldsymbol{\Phi}}_{I} \mathbf{u}_{I}\right)^{T} \mathbf{S}\left(\sum_{J=1}^{n} \widetilde{\boldsymbol{\Phi}}_{J} \mathbf{u}_{J}-\overline{\mathbf{u}}\right) d \Gamma .
\end{aligned}
$$

The discrete form is

$$
\alpha \int_{\Gamma_{u}} \delta \mathbf{u}^{T} \mathbf{S}(\mathbf{u}-\overline{\mathbf{u}}) d \Gamma=\mathbf{K}^{\alpha} \mathbf{U}-\mathbf{F}^{\alpha},
$$

where

$$
\begin{gathered}
\mathbf{K}_{I J}=\alpha \int_{\Gamma_{u}} \widetilde{\boldsymbol{\Phi}}_{I}^{T} \cdot \mathbf{S} \cdot \widetilde{\boldsymbol{\Phi}}_{J} d \Gamma, \\
\mathbf{F}_{I}^{\alpha}=\alpha \int_{\Gamma_{u}} \widetilde{\boldsymbol{\Phi}}_{I}^{T} \cdot \mathbf{S} \cdot \overline{\mathbf{u}} d \Gamma .
\end{gathered}
$$

From (28) and discrete forms (26) and (31), the final equation of system can be obtained as

$$
\mathbf{M U ̈}(t)+\mathbf{C} \dot{\mathbf{U}}(t)+\left(\mathbf{K}+\mathbf{K}^{\alpha}\right) \mathbf{U}(t)=\mathbf{F}(t)+\mathbf{F}^{\alpha} .
$$

Let

$$
\begin{aligned}
\overline{\mathbf{K}} & =\mathbf{K}+\mathbf{K}^{\alpha}, \\
\overline{\mathbf{F}}(t) & =\mathbf{F}(t)+\mathbf{F}^{\alpha} .
\end{aligned}
$$

Then (33) can be simplified as

$$
\mathbf{M} \ddot{\mathbf{U}}(t)+\mathbf{C} \dot{\mathbf{U}}(t)+\overline{\mathbf{K}} \mathbf{U}(t)=\overline{\mathbf{F}}(t) .
$$

If the damping is neglected, the equation of system can be simplified as

$$
\mathbf{M} \ddot{\mathbf{U}}(t)+\overline{\mathbf{K}} \mathbf{U}(t)=\overline{\mathbf{F}}(t) .
$$

If the right side of (36) is equal to zero, the expression is a free vibration equation of the system.

\section{Time Integration Scheme}

In the dynamic analysis, due to the inertia force and damping force appearing in the equilibrium equation, the mass matrix and damping matrix are introduced to the final solving equation, which makes the system discrete equation not algebraic equations, but ordinary differential equations.

For the two order ordinary differential equations, the solutions can be generally obtained by using the method of solving ordinary differential equations. However, in dynamic analysis of the meshless manifold method, the above method is not efficient because the order of the matrix is very high. The common method is the direct integral method and the mode superposition method in which the two methods are essentially equivalent. From the computational efficiency, the direct integral method is used in this paper.

There are two direct integration methods which are central difference method and Newmark method. The central difference method is a stable conditional algorithm and the time step $\Delta t$ must be less than a certain critical value $\Delta t_{\mathrm{cr}}$ which is decided by the solving problem; otherwise the algorithm will be unstable. Therefore, the central difference method is suitable for solving the problem of wave propagation, and not suitable for the structural dynamics. Because the low frequency component of the dynamic response of the structure is the main, a larger time step may allow to using from the calculation accuracy, and not necessary to limit the time step to smaller. As a result, a nonconditional stable implicit algorithm for the structural dynamics, which is mainly determined by the accuracy requirements, is usually adopted. The Newmark method is used in this paper. 
The time domain $0 \rightarrow T$ is divided into $n$ time intervals, $\Delta t(=T / n)$. The specific calculation is that a time interval is moving forward step by step; calculation of the next time is based on the previous time step. If the solutions of the moment of $0, \Delta t, 2 \Delta t, \ldots, t$ are obtained, the next computation is to solve the solution of the moment of $t+\Delta t$.

Newmark integral method is essentially an extension of linear acceleration method. In the time step of $t \rightarrow t+\Delta t$, the recursive relation of displacement and velocity is

$$
\begin{aligned}
\mathbf{U}_{t+\Delta t}= & \mathbf{U}_{t}+\Delta t \dot{\mathbf{U}}_{t}+\frac{1}{2}\left(1-\beta_{2}\right) \Delta t^{2} \ddot{\mathbf{U}}_{t} \\
& +\frac{1}{2} \beta_{2} \Delta t^{2} \ddot{\mathbf{U}}_{t+\Delta t}, \\
\dot{\mathbf{U}}_{t+\Delta t}= & \dot{\mathbf{U}}_{t}+\left(1-\beta_{1}\right) \Delta t \ddot{\mathbf{U}}_{t}+\beta_{1} \Delta t \ddot{\mathbf{U}}_{t+\Delta t},
\end{aligned}
$$

where parameters of $\beta_{1}$ and $\beta_{2}$ are related to the stability and accuracy of the results. Different parameters are chosen to correspond to different integral methods.

Linear acceleration method is as follows:

$$
\begin{aligned}
& \beta_{1}=\frac{1}{2}, \\
& \beta_{2}=\frac{1}{3} .
\end{aligned}
$$

Averaged acceleration method is as follows:

$$
\begin{aligned}
& \beta_{1}=\frac{1}{2}, \\
& \beta_{2}=\frac{1}{2} .
\end{aligned}
$$

Uniform acceleration method is as follows:

$$
\begin{aligned}
& \beta_{1}=\frac{1}{2}, \\
& \beta_{2}=0 .
\end{aligned}
$$

Galerkin method is as follows:

$$
\begin{aligned}
& \beta_{1}=\frac{3}{2}, \\
& \beta_{2}=\frac{8}{5} .
\end{aligned}
$$

Backward difference method is as follows:

$$
\begin{aligned}
& \beta_{1}=\frac{3}{2}, \\
& \beta_{2}=4 .
\end{aligned}
$$

From (37), we have

$$
\begin{aligned}
\ddot{\mathbf{U}}_{t+\Delta t}= & \frac{2}{\beta_{2} \Delta t^{2}}\left(\mathbf{U}_{t+\Delta t}-\mathbf{U}_{t}\right)-\frac{2}{\beta_{2} \Delta t} \dot{\mathbf{U}}_{t} \\
& -\left(\frac{1}{\beta_{2}}-1\right) \ddot{\mathbf{U}}_{t} .
\end{aligned}
$$

Let

$$
\begin{aligned}
& \alpha_{1}=\frac{2}{\beta_{2} \Delta t^{2}}, \\
& \alpha_{2}=\frac{2}{\beta_{2} \Delta t}, \\
& \alpha_{3}=\frac{1}{\beta_{2}}-1 .
\end{aligned}
$$

Equation (44) can be written as

$$
\ddot{\mathbf{U}}_{t+\Delta t}=\alpha_{1}\left(\mathbf{U}_{t+\Delta t}-\mathbf{U}_{t}\right)-\alpha_{2} \dot{\mathbf{U}}_{t}-\alpha_{3} \ddot{\mathbf{U}}_{t} .
$$

Substituting (46) into (38), we have

$$
\begin{aligned}
\dot{\mathbf{U}}_{t+\Delta t}= & \beta_{1} \alpha_{2}\left(\mathbf{U}_{t+\Delta t}-\mathbf{U}_{t}\right)+\left(1-\frac{2 \beta_{1}}{\beta_{2}}\right) \dot{\mathbf{U}}_{t} \\
& +\left(1-\frac{\beta_{1}}{\beta_{2}}\right) \Delta t \ddot{\mathbf{U}}_{t} .
\end{aligned}
$$

In the Newmark method, the displacement solution $\mathbf{U}_{t+\Delta t}$ at time of $t+\Delta t$ is obtained by solving (35); that is,

$$
\mathbf{M} \ddot{\mathbf{U}}_{t+\Delta t}+\mathbf{C} \dot{\mathbf{U}}_{t+\Delta t}+\overline{\mathbf{K}} \mathbf{U}_{t+\Delta t}=\overline{\mathbf{F}}_{t+\Delta t} .
$$

Substituting (46) and (47) into (48), we have

$$
\begin{aligned}
& \left(\alpha_{1} \mathbf{M}+\beta_{1} \alpha_{2} \mathbf{C}+\overline{\mathbf{K}}\right) \mathbf{U}_{t+\Delta t}=\overline{\mathbf{F}}_{t+\Delta t} \\
& \quad+\mathbf{M}\left(\alpha_{1} \mathbf{U}_{t}+\alpha_{2} \dot{\mathbf{U}}_{t}+\alpha_{3} \ddot{\mathbf{U}}_{t}\right) \\
& \quad+\mathbf{C}\left[\beta_{1} \alpha_{2} \mathbf{U}_{t}+\left(\frac{2 \beta_{1}}{\beta_{2}}-1\right) \dot{\mathbf{U}}_{t}+\left(\frac{\beta_{1}}{\beta_{2}}-1\right) \Delta t \ddot{\mathbf{U}}_{t}\right] .
\end{aligned}
$$

After $\mathbf{U}_{t+\Delta t}$ being calculated, $\dot{\mathbf{U}}_{t+\Delta t}$ and $\ddot{\mathbf{U}}_{t+\Delta t}$ can be obtained by (47) and (46), respectively.

\section{Numerical Example}

The two-dimensional rectangular plate's width is $2 \mathrm{~m}$ and length is $4 \mathrm{~m}$; the free end is subjected to a suddenly applied uniform distributed load, as shown in Figure 1. Modulus of elasticity is $E=80 \mathrm{Gpa}$, Poisson ratio is $\nu=0.25$, and mass density is $\rho=2450 \mathrm{~kg} / \mathrm{m}^{3}$, without consideration of the influence of damping.

At the moment $t=\mathbf{0}$. The free end of the beam is subjected to a suddenly applied uniform distributed load $P(t)=1 \mathrm{kN} / \mathrm{m}^{2}$, as shown in Figure 2 .

Initial condition for the structure is $\mathbf{u}_{0}=\dot{\mathbf{u}}_{0}=0$, the analytical solution of the beam can be obtained by using the mode superposition method, and the axial displacement is

$$
\begin{aligned}
& u(x, t)=\frac{8 P L}{\pi^{2} E A} \\
& \cdot \sum_{n=1}^{\infty}\left\{\frac{(-1)^{n-1}}{(2 n-1)^{2}} \sin \frac{(2 n-1) \pi x}{2 L}\left(1-\cos \omega_{n} t\right)\right\} .
\end{aligned}
$$




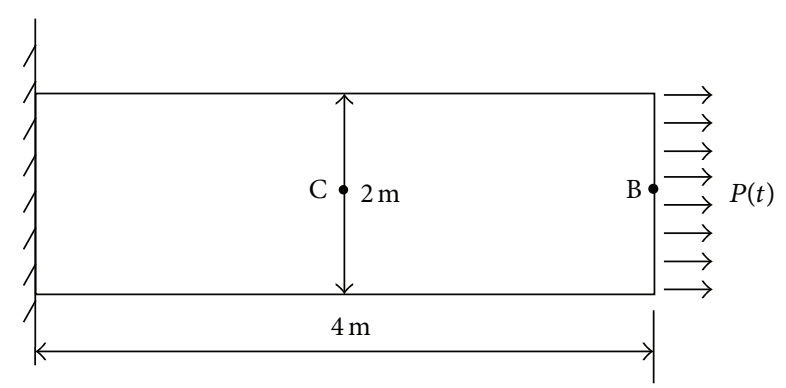

FIGURE 1: The cantilever beam subjected to a suddenly applied uniform distributed load at free end.

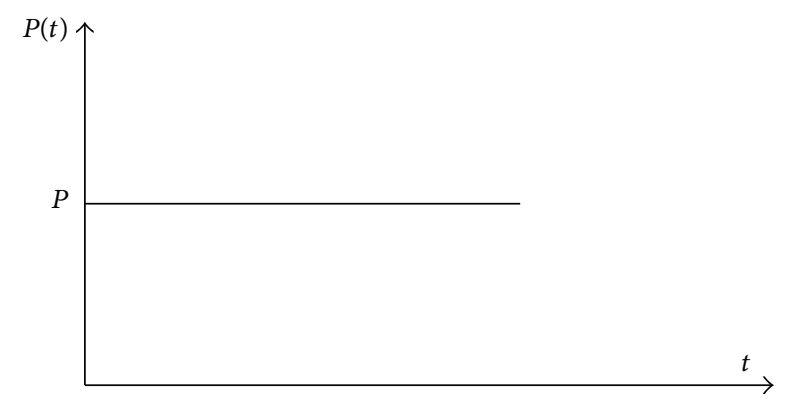

FIgURE 2: The change curve of the suddenly applied load with time.

The axial stress is

$$
\begin{aligned}
& \sigma(x, t)=E \frac{\partial u(x, t)}{\partial x} \\
& =\frac{4 P}{\pi A} \sum_{n=1}^{\infty}\left\{\frac{(-1)^{n-1}}{(2 n-1)} \cos \frac{(2 n-1) \pi x}{2 L}\left(1-\cos \omega_{n} t\right)\right\},
\end{aligned}
$$

where $\omega_{n}$ is frequency, which is $\omega_{n}=(2 n-1) \pi a /(2 L), L$ is length of plate, $A$ is the cross-sectional area of plate, $a=$ $\sqrt{E / \rho}$, and $P$ is the exciting force acting on the right side of the plate, which is $P=P(t)$.

In this example, the complex variable meshless manifold method is used to discrete the spatial domain and together with the Newmark time integration method is used to simulate the dynamics. We obtain the change of the displacement and stress of the point $B$ at the free end versus time and the change of the displacement and stress of the central point $\mathrm{C}$ versus time. The numerical results are compared with the analytical solutions.

The distributions of regular nodes of $20 \times 40$ are adopted, as shown in Figure 3. The linear basis function and Gauss weighted function are used in analysis, and the backward difference method ( $\gamma=3 / 2, \beta=2)$ of Newmark method is used in dynamic simulation; the time step is $\Delta t=0.0001 \mathrm{~s}$.

The relationships between the displacement of points $B$ and $C$ versus time are shown in Figures 4 and 5, the relationships between the stress of points $\mathrm{B}$ and $\mathrm{C}$ are given in Figures 6 and 7, respectively, and the numerical results are compared with the analytical solutions. From Figures 47 , the numerical solutions are in good agreement with the

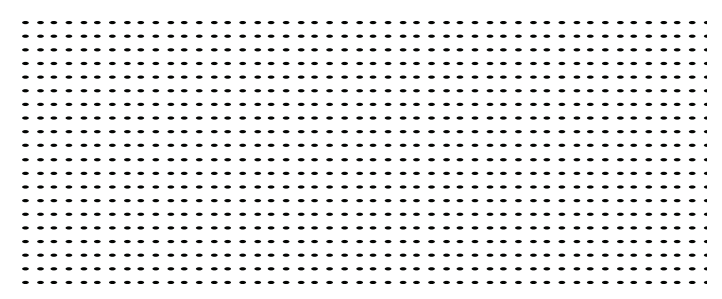

FIGURE 3: Distribution of nodes.

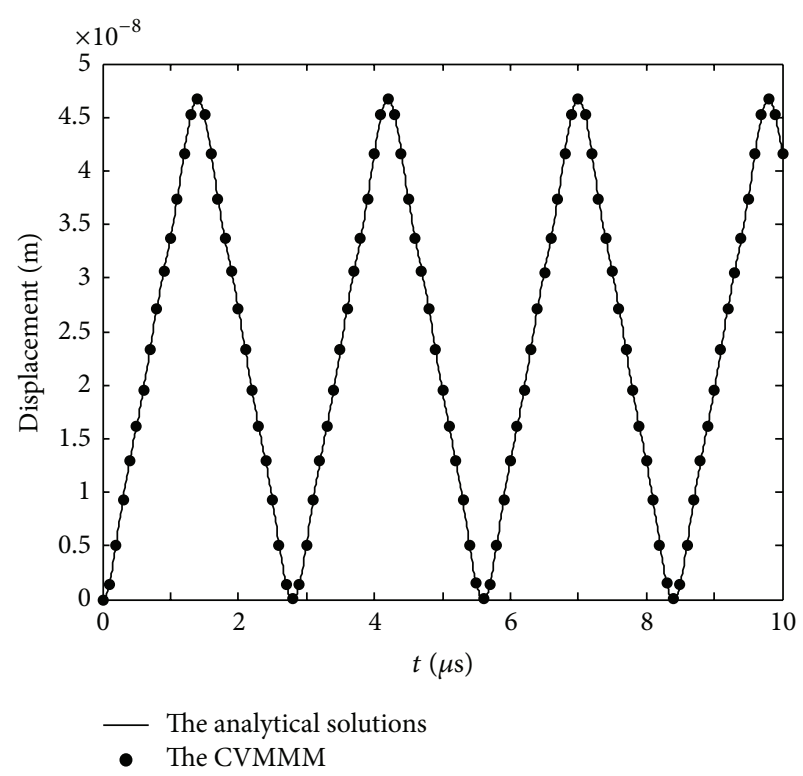

FIgURE 4: Comparison of the numerical and the analytical solutions of displacement versus time at point B.

analytical solutions. The results show that the CVMMM and the discrete forms are correct and the method is feasible. The figures show that the Newmark method does not have the ability to dissipate the high frequency, so the stresses have a dramatic oscillation phenomenon.

\section{Conclusion}

In this paper, the complex variable meshless method is applied to solve the problem of elastic dynamics, the complex variable meshless manifold method for dynamics is established, and the corresponding formula is derived. The numerical example shows that the numerical solutions are in good agreement with the analytical solution; the results illustrate that the CVMMM and the discrete forms are correct and the method is feasible. Compared with the traditional meshless manifold method, the CVMMM has higher accuracy in the same distribution of nodes.

Because finite covering technology of manifold analysis is used, the CVMMM can handle the domain of continuous and discontinuous problems and can overcome the mapping 


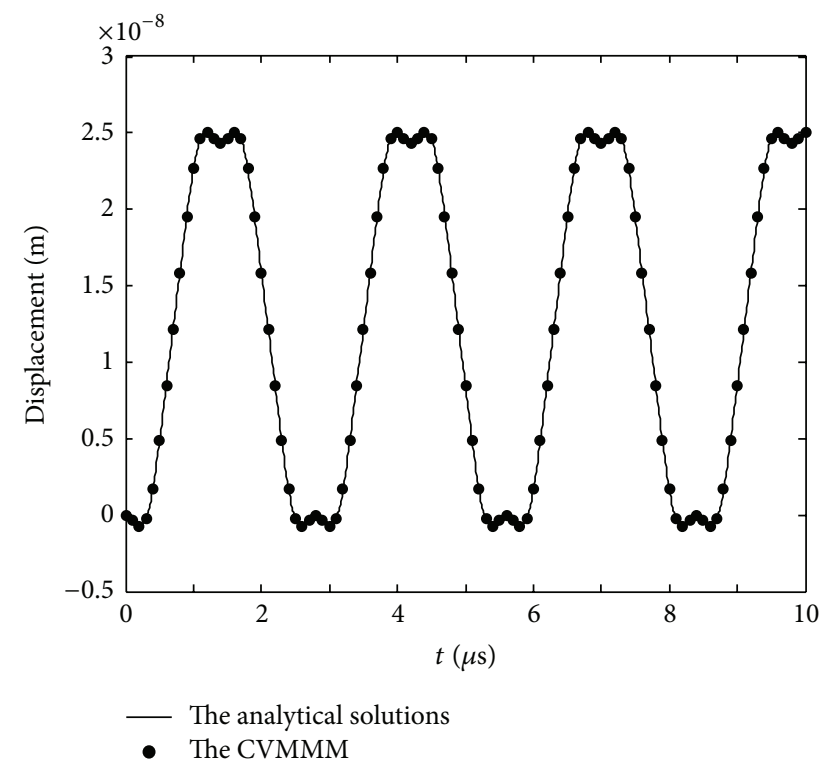

FIGURE 5: Comparison of the numerical and the analytical solutions of displacement versus time at point $\mathrm{C}$.

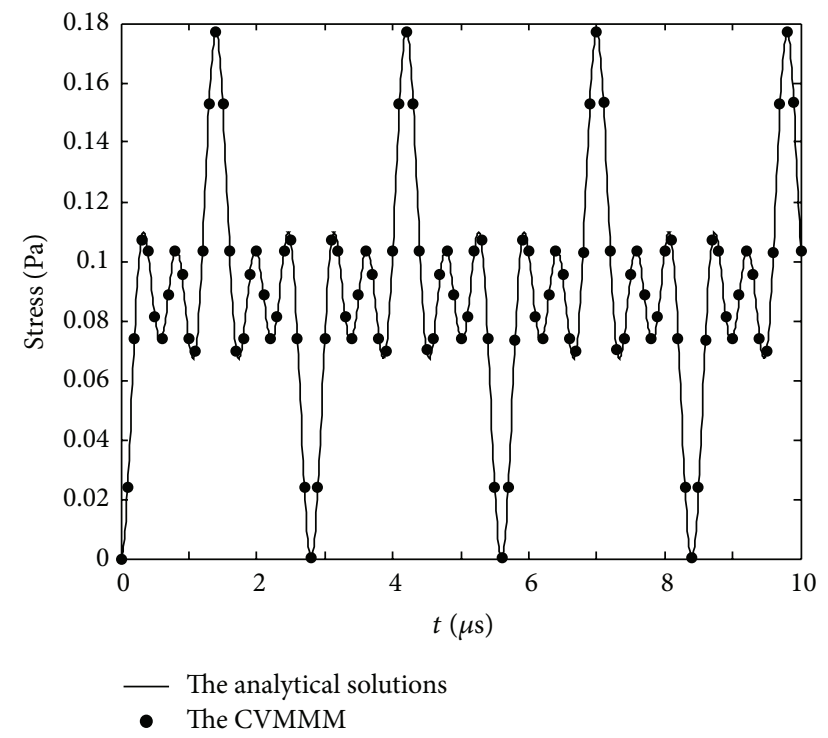

FIGURE 6: Comparison of the numerical and the analytical solutions of stress versus time at point $B$.

process of long crack in finite element method or meshless method. The accuracy of numerical solution is improved.

The CVMMM can be applied to the problems such as crack propagation or physical nonlinearity and geometric nonlinearity and can be further used to solve practical engineering problems.

\section{Competing Interests}

The authors declare that they have no competing interests.

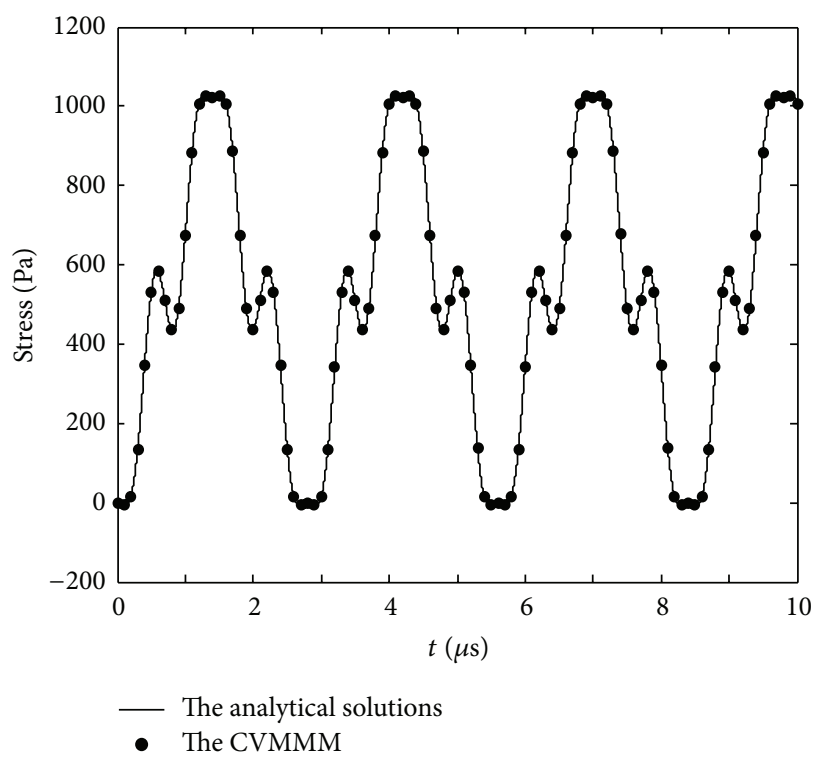

FIGURE 7: Comparison of the numerical and the analytical solutions of stress versus time at point $C$.

\section{Acknowledgments}

The work is supported by the National Natural Science Foundation of China through Grant 11271234 and the Science Program of Shandong Provincial Education Department through Grant J13LJ51.

\section{References}

[1] K. Arakawa, T. Mada, and K. Takahashi, "Correlations among dynamic stress intensity factor, crack velocity and acceleration in brittle fracture," International Journal of Fracture, vol. 105, no. 4, pp. 311-320, 2000.

[2] J.-B. Leblond and P. Mouro, "Crack propagation from a preexisting flaw at a notch root. I. Introduction and general form of the stress intensity factors at the initial crack tip," International Journal of Fracture, vol. 104, no. 3, pp. 211-224, 2000.

[3] M. L. Wilkins, "Numerical analysis of dynamic crack problems," in Mechanics of Fracture, G. C. Sih, Ed., vol. 4, P. Noordhoff, Leyden, Netherlands, 1977.

[4] M. H. Aliabadi, "Boundary element formulations in fracture mechanics," Applied Mechanics Reviews, vol. 50, no. 2, pp. 8396, 1997.

[5] J. Sládek and V. Sládek, "Dynamic stress intensity factors studied by boundary integro-differential equations," International Journal for Numerical Methods in Engineering, vol. 23, no. 5, pp. 919928, 1986.

[6] P. Fedeliński, "Boundary element method in dynamic analysis of structures with cracks," Engineering Analysis with Boundary Elements, vol. 28, no. 9, pp. 1135-1147, 2004.

[7] O. C. Zienkiewicz, The Finite Element Method, McGraw-Hill, 3rd edition, 1977.

[8] C. A. Brebbia, The Boundary Element Method for Engineers, Pentech Press, London, UK, 1978.

[9] L. B. Lucy, "A numerical approach to the testing of the fission hypothesis," The Astronomical Journal, vol. 8, no. 12, pp. 10131024, 1977. 
[10] R. A. Gingold and J. J. Moraghan, "Smoothed particle hydrodynamics-theory and application to non-spherical stars," Monthly Notices of the Royal Astronomical Society, vol. 181, pp. 375-389, 1977.

[11] T. Belytschko, Y. Y. Lu, and L. Gu, "Element-free Galerkin methods," International Journal for Numerical Methods in Engineering, vol. 37, no. 2, pp. 229-256, 1994.

[12] H.-J. Chung and T. Belytschko, "An error estimate in the EFG method," Computational Mechanics, vol. 21, no. 2, pp. 91-100, 1998.

[13] E. Oñate, S. Idelsohn, O. C. Zienkiewicz, and R. L. Taylor, "A finite point method in computational mechanics. Applications to convective transport and fluid flow," International Journal for Numerical Methods in Engineering, vol. 39, no. 22, pp. 38393866, 1996.

[14] E. Onate and S. Idelsohn, "A mesh-free finite point method for advective-diffusive transport and fluid flow problems," Computational Mechanics, vol. 21, no. 4-5, pp. 283-292, 1998.

[15] H. Fatahi, J. Saberi-Nadjafi, and E. Shivanian, "A new spectral meshless radial point interpolation (SMRPI) method for the two-dimensional Fredholm integral equations on general domains with error analysis," Journal of Computational and Applied Mathematics, vol. 294, pp. 196-209, 2016.

[16] W. K. Liu, S. Jun, and Y. F. Zhang, "Reproducing kernel particle methods," International Journal for Numerical Methods in Fluids, vol. 20, no. 8-9, pp. 1081-1106, 1995.

[17] W. K. Liu, S. Jun, D. T. Sihling, Y. Chen, and W. Hao, "Multiresolution reproducing kernel particle methods for computational fluid dynamics," International Journal for Numerical Methods in Fluid, vol. 24, no. 12, pp. 1391-1415, 1997.

[18] C. A. Duarte and J. T. Oden, "H-p clouds-an $h-p$ meshless method," Numerical Methods for Partial Differential Equations, vol. 12, no. 6, pp. 673-705, 1996.

[19] J. M. Melenk and I. Babuška, "The partition of unity finite element method: basic theory and applications," Computer Methods in Applied Mechanics and Engineering, vol. 139, no. 1-4, pp. 289-314, 1996.

[20] Y. C. Cai, L. Han, L. G. Tian, and L. Y. Zhang, "Meshless method based on Shepard function and partition of unity for two-dimensional crack problems," Engineering Analysis with Boundary Elements, vol. 65, pp. 126-135, 2016.

[21] J. T. Oden, A. Duarte, and O. C. Zienkiewicz, "A new cloudbased hp finite element method," International Journal for Numerical Methods in Engineering, vol. 50, pp. 160-170, 1998.

[22] S. N. Atluri and T. Zhu, "A new meshless local Petrov-Galerkin (MLPG) approach in computational mechanics," Computational Mechanics, vol. 22, no. 2, pp. 117-127, 1998.

[23] X. Zhang, X.-H. Liu, K.-Z. Song, and M.-W. Lu, "Leastsquares collocation meshless method," International Journal for Numerical Methods in Engineering, vol. 51, no. 9, pp. 1089-1100, 2001.

[24] K. M. Liew, J. Ren, and S. Kitipornchai, "Analysis of the pseudoelastic behavior of a SMA beam by the element-free Galerkin method," Engineering Analysis with Boundary Elements, vol. 28, no. 5, pp. 497-507, 2004.

[25] Y. M. Cheng and J. H. Li, "A meshless method with complex variables for elasticity," Acta Physica Sinica, vol. 54, no. 10, pp. 4463-4471, 2005.

[26] Y. J. Deng, C. Liu, M. J. Peng, and Y. M. Cheng, "The interpolating complex variable element-free galerkin method for temperature field problems," International Journal of Applied Mechanics, vol. 7, no. 2, Article ID 1550017, 2015.
[27] H. F. Gao and Y. M. Cheng, "A complex variable meshless manifold method for elasticity problems," Acta Mechanica Sinica, vol. 41, no. 3, pp. 1-9, 2009.

[28] K. M. Liew, C. Feng, Y. Cheng, and S. Kitipornchai, "Complex variable moving least-squares method: a meshless approximation technique," International Journal for Numerical Methods in Engineering, vol. 70, no. 1, pp. 46-70, 2007.

[29] F. X. Sun, J. F. Wang, Y. M. Cheng, and A. X. Huang, "Error estimates for the interpolating moving least-squares method in n-dimensional space," Applied Numerical Mathematics, vol. 98, pp. 79-105, 2015.

[30] H. F. Gao and Y. M. Cheng, "A complex variable meshless manifold method for fracture problems," International Journal of Computational Methods, vol. 7, no. 1, pp. 55-81, 2010.

[31] H. F. Gao and G. F. Wei, "Stress intensity factor for interface cracks in bimaterials using complex variable meshless manifold method," Mathematical Problems in Engineering, vol. 2014, Article ID 353472, 8 pages, 2014. 


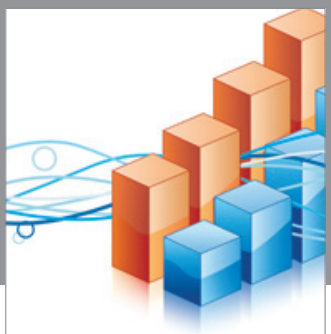

Advances in

Operations Research

vatem alat4

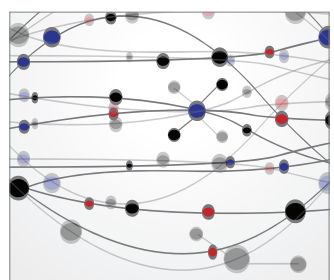

\section{The Scientific} World Journal
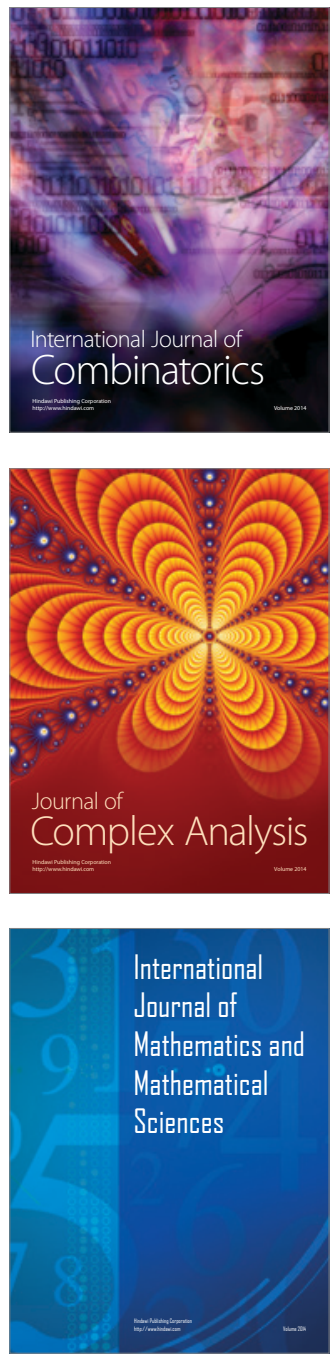
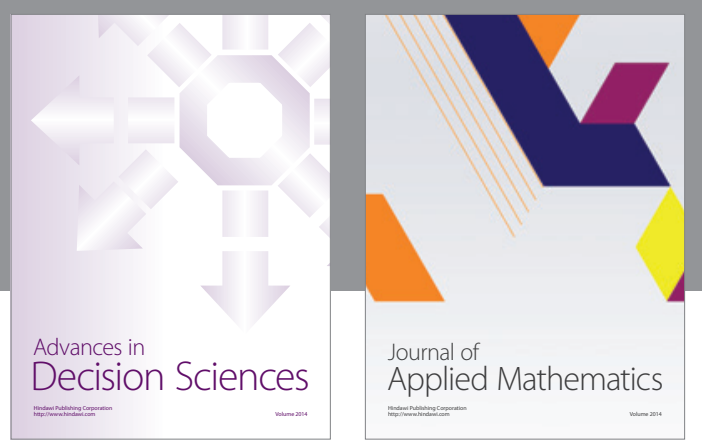

Algebra

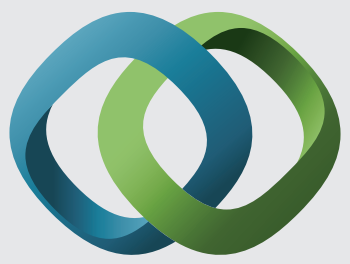

\section{Hindawi}

Submit your manuscripts at

http://www.hindawi.com
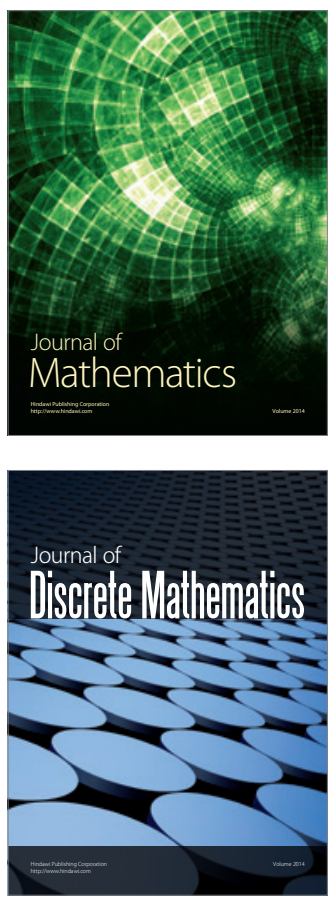

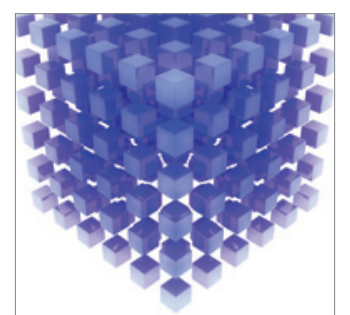

Mathematical Problems in Engineering
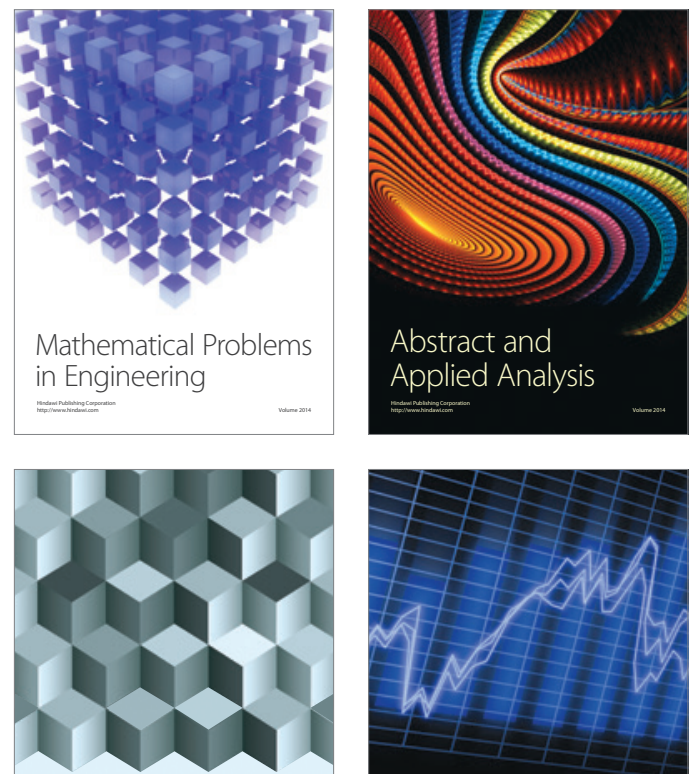

Journal of

Function Spaces

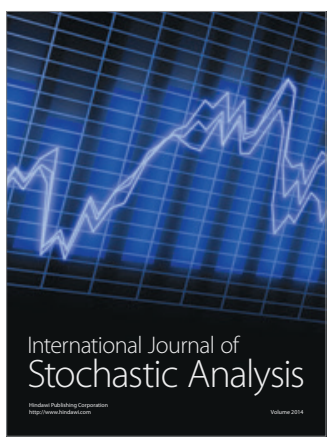

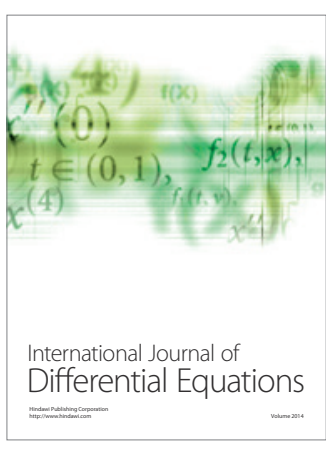
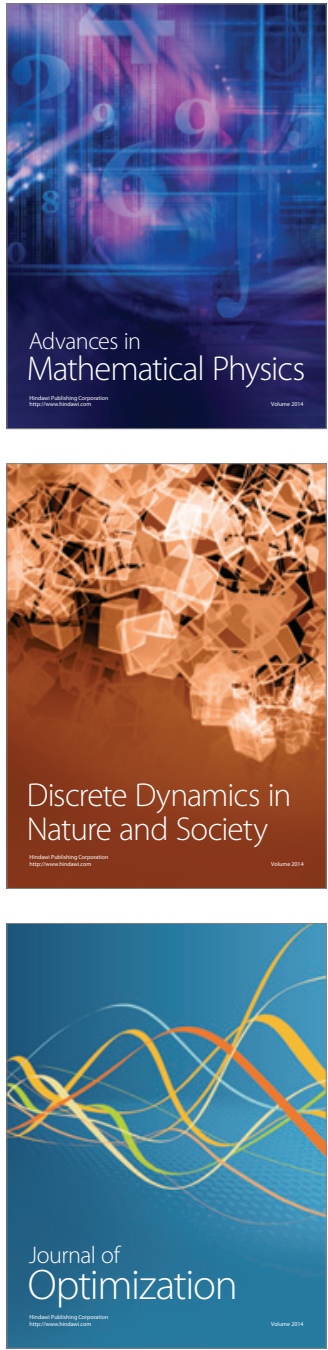